\title{
On Carbon Star Evolution in the IRAS Two-Color Diagram
}

\section{RYSZARD SZCZERBA ${ }^{1,3}$, MATTHIAS STEFFEN ${ }^{2}$, and KEVIN VOLK ${ }^{3}$}

\author{
${ }^{1}$ Nicolaus Copernicus Astronomical Center, Toruń, Poland \\ ${ }^{2}$ Astrophysikalisches Institut Potsdam, Germany \\ 3 The University of Calgary, Calgary, Alberta, Canada
}

We present a comparison between recent stellar evolutionary calculations by Blöcker $(1995, A \& A, 297,727)$ and by Vassiliadis \& Wood $(1993, A p J$, 413,641 ) based on the properties of the circumstellar shells predicted by these two sets of models, which mainly differ in the mass-loss rates adopted during evolution on the Asymptotic Giant Branch (AGB). Given the mass-loss rate as a function of time, the evolution of the dusty circumstellar shell and of the resulting synthetic spectrum is computed with a time-dependent radiation hydrodynamics code (see Steffen et al., this volume). Since the formation and further evolution of $\mathrm{C}$ stars is a strong function of mass loss on the AGB (Bryan et al. 1990, $A p J, 365,301$; Groenewegen \& de Jong 1993, A\&A, 267, 410 ) it is possible to examine different scenarios for the mass-loss behavior by comparing their predictions to different observational characteristics of AGB stars. We discuss the evolution in the IRAS two-color diagram, from just before the moment of carbon star formation until the end of their evolution on the AGB. We have adopted the widely accepted scenario that carbon stars are formed during thermal pulses but we have tested different assumptions concerning the moment of their formation (core mass, thermal pulse number).

It has been suggested that visual carbon stars represent a period when mass loss is interrupted. According to both mass-loss scenarios mentioned above, the mass-loss rate is strongly modulated by thermal pulses on the upper AGB, leading to a repeating cycle of evolution from visual to infrared carbon stars after the formation of a C-rich star from an O-rich one. This cyclic variability of mass loss, which previous simulations have not taken into account, explains in a natural way the existence of visual carbon stars with O-rich as well as with only C-rich shells. It also explains why the fraction of visual carbon stars is higher than predicted if the star becomes a visual carbon star only once in its evolution. 